BNL-112153-2016-NE

\title{
Fishing for isotopes in the Brookhaven Lab Isotope Producer (BLIP) cooling water
}

\author{
Jonathan Fitzsimmons
}

April 2016

Collider Accelerator Department

Brookhaven National Laboratory

\author{
U.S. Department of Energy \\ USDOE Office of Science (SC), \\ Nuclear Physics (NP) (SC-26)
}

Notice: This manuscript has been authored by employees of Brookhaven Science Associates, LLC under Contract No. DE-SC0012704 with the U.S. Department of Energy. The publisher by accepting the manuscript for publication acknowledges that the United States Government retains a non-exclusive, paid-up, irrevocable, world-wide license to publish or reproduce the published form of this manuscript, or allow others to do so, for United States Government purposes. 


\section{DISCLAIMER}

This report was prepared as an account of work sponsored by an agency of the United States Government. Neither the United States Government nor any agency thereof, nor any of their employees, nor any of their contractors, subcontractors, or their employees, makes any warranty, express or implied, or assumes any legal liability or responsibility for the accuracy, completeness, or any third party's use or the results of such use of any information, apparatus, product, or process disclosed, or represents that its use would not infringe privately owned rights. Reference herein to any specific commercial product, process, or service by trade name, trademark, manufacturer, or otherwise, does not necessarily constitute or imply its endorsement, recommendation, or favoring by the United States Government or any agency thereof or its contractors or subcontractors. The views and opinions of authors expressed herein do not necessarily state or reflect those of the United States Government or any agency thereof. 


\section{Fishing for isotopes in the Brookhaven Lab Isotope Producer (BLIP) cooling water.}

Be-7 has been used in environmental studies; the isotope is produced during BLIP irradiations and accumulates in the 320 gallons of cooling water. Be-7 has a 53.24 day half-life, so the optimal production/purification time is at the end of the BLIP run season. To purify Be-7 fifteen to twenty gallons of BLIP cooling water are removed and pumped through ion exchange columns that retain Be-7. This labor intensive approach captures $\sim 15 \mathrm{mCi}$ of Be-7, but the solution requires further purification. The method can lead to increased radiation exposure to staff.

The ideal way to capture isotopes from large volumes is to reach in to the solution and selectively pull out the desired isotope. It is a lot like fishing. In junior high school I would go to a neighbor's pond and test various fishing flies I made to see what would catch fish. On one trip the "monstrous" largemouth bass was in the shallow section of the pond, and I would frantically attempt to catch the 20+ inch fish. I would change flies repeatedly and drop the fly around the fish, slowly moving the fly by the large fish in hopes of a bite. The importance of using the right bait in fishing became clear. One approach to simplify the process to capture Be-7 from BLIP is to add solid supports (17x6 mm) with metal capturing groups to the BLIP cooling water, let the pumps circulate the water and attempt to capture Be-7. This would remove the need to remove large volumes of water and would be less labor intensive. A 3D printed plastic target box was built that fit in the target holder at BLIP, which could hold hundreds of solid supports and allow BLIP cooling water to flow through the box (Figure 1). The first experiment was to determine the best "bait" to capture Be-7. Solid supports containing the following metal capturing groups were used: iminodiacetate, sulfonic acid, quaternary 
amine, pyridine amine, pyridine amine acid. The different solid supports were tagged with different color stems, placed in the target box and placed in the BLIP cooling water for 1 week. One sulfonic acid solid support was able to capture $\sim 2 \mathrm{mCi}$ of Be-7 with $~ 93$ $\%$ radiopurity, and each of the other solid supports captured less than $0.1 \mathrm{mCi}$. Release of Be-7 from the solid support with $10 \mathrm{M} \mathrm{HCl}$ occurs within 2 minutes, and the Be-7 solution can be purified to DOE specifications by passing the solution through an anion exchange column (product radiopurity $99.5 \%$, chemical recovery 85\%).

In fly fishing if one matches the hatch and uses the correct bait and technique catching fish becomes fun and simple. On one occasion I caught seven fish on seven consecutive casts and "retired" the fly. For capturing Be-7 from the cooling water we have done an uptake study with the sulfonic acid solid support and found the uptake is linear over time ( $\mathrm{y}=4.11 \mathrm{X}, \mathrm{R}^{2}=0.99$ ) and $98 \%$ uptake occurs within 24 hours. To capture large amounts of Be-7 we have used 38 sulfonic acid solid supports and were able to capture $65 \mathrm{mCi}$ of Be-7 in 24 hours. During the experiments the surface color of the iminodiacetate and sulfonic acid solid supports changed from yellow to blue-green (Figure 2). The solid supports could be reused multiple times to capture more Be-7. The experiment proved the solid supports can be used to capture isotopes from large bodies of water and could be used in radioactive waste treatment or cleanup of metal contaminated pools. Special thanks to Lisa Muench, Christian Cullen and Jason Nalepa for performing the work.

Jonathan Fitzsimmons

The Irish and their fishing stories: in the end I did catch the largemouth bass that day, but not the way you think, so I release the fish back into the pond and never caught it again. 


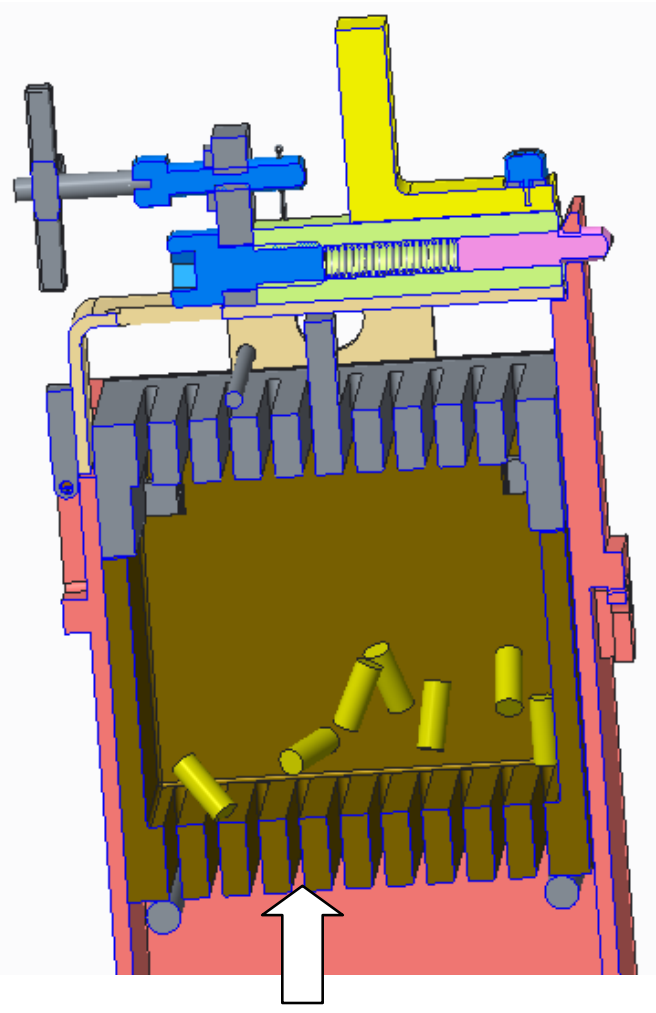

Flow of BLIP cooling water

Figure 1. A cross sectional view of the target box assembly used to capture Be-7 from the BLIP cooling water. The yellow cylinders represent the sulfonic acid solid supports.

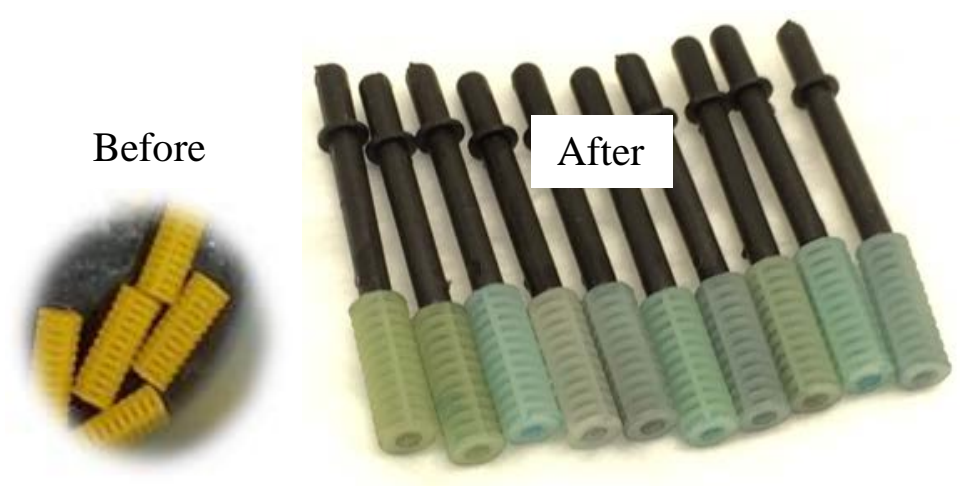

Figure 2. Solid supports used to capture Be-7 in BLIP cooling water. Analysis indicated the 38 sulfonic acid solid supports captured $65 \mathrm{mCi}$ of Be-7 (14\% of total Be-7 in the BLIP tank water), $30 \mathrm{mg}$ of $\mathrm{Cu}$ (5.6\%), $5 \mathrm{mg}$ of $\mathrm{Mn}$ (5.5\%), and $23 \mathrm{mg}$ of $\mathrm{Ni}$ (5.9\%). 